Open Access

\title{
Efficacy and safety of patisiran for familial amyloidotic polyneuropathy: a phase II multi-dose study
}

Ole B Suhr ${ }^{1 *}$, Teresa Coelho ${ }^{2}$, Juan Buades ${ }^{3}$, Jean Pouget ${ }^{4}$, Isabel Conceicao ${ }^{5}$, John Berk ${ }^{6}$, Hartmut Schmidt ${ }^{7}$, Márcia Waddington-Cruz ${ }^{8}$, Josep M. Campistol ${ }^{9}$, Brian R. Bettencourt ${ }^{10}$, Akshay Vaishnaw $^{10}$, Jared Gollob ${ }^{10}$ and David Adams ${ }^{11}$

\begin{abstract}
Background: Transthyretin-mediated amyloidosis is an inherited, progressively debilitating disease caused by mutations in the transthyretin gene. This study evaluated the safety, tolerability, pharmacokinetics, and pharmacodynamics of multiple doses of patisiran (ALN-TTR02), a small interfering RNA encapsulated within lipid nanoparticles, in patients with transthyretin-mediated familial amyloid polyneuropathy (FAP).

Methods: In this phase II study, patients with FAP were administered 2 intravenous infusions of patisiran at one of the following doses: $0.01(n=4), 0.05(n=3), 0.15(n=3)$, or $0.3(n=7) \mathrm{mg} / \mathrm{kg}$ every 4 weeks (Q4W), or $0.3 \mathrm{mg} / \mathrm{kg}(n=12)$ every 3 weeks (Q3W).

Results: Of 29 patients in the intent-to-treat population, 26 completed the study. Administration of patisiran led to rapid, dose-dependent, and durable knockdown of transthyretin, with the maximum effect seen with patisiran $0.3 \mathrm{mg} / \mathrm{kg}$; levels of mutant and wild-type transthyretin were reduced to a similar extent in Val30Met patients. A mean level of knockdown exceeding $85 \%$ after the second dose, with maximum knockdown of $96 \%$, was observed for the Q3W dose. The most common treatment-related adverse event (AE) was mild-to-moderate infusion-related reactions in $10.3 \%$ of patients. Four serious AEs (SAEs) were reported in 1 patient administered $0.3 \mathrm{mg} / \mathrm{kg}$ Q $3 \mathrm{~W}$ (urinary tract infection, sepsis, nausea, vomiting), and 1 patient administered $0.3 \mathrm{mg} / \mathrm{kg}$ Q $4 \mathrm{~W}$ had 1 SAE (extravasation-related cellulitis).
\end{abstract}

Conclusions: Patisiran was generally well tolerated and resulted in significant dose-dependent knockdown of transthyretin protein in patients with FAP. Patisiran $0.3 \mathrm{mg} / \mathrm{kg}$ Q3W is currently in phase III development.

Trial registration number: NCT01617967.

Keywords: Patisiran, RNA interference, Transthyretin-mediated familial amyloidotic polyneuropathy, Polyneuropathy, Hereditary disease, Genetic mutation, Phase II, Clinical trial

\section{Background}

Transthyretin (TTR) is a tetrameric protein produced primarily in the liver. Mutations in the TTR gene destabilize the tetramer, leading to misfolding of monomers and aggregation into TTR amyloid fibrils (ATTR). Tissue deposition results in systemic ATTR amyloidosis

\footnotetext{
* Correspondence: Ole.suhr@medicin.umu.se

'Department of Public Health and Clinical Medicine, Umeå University, 90187 Umeå, Sweden

Full list of author information is available at the end of the article
}

[1-3]. Over 100 reported TTR mutations exhibit a spectrum of disease symptoms. The most common mutations associated with familial amyloid polyneuropathy (FAP) and ATTR-associated cardiomyopathy, respectively, are Val30Met [4] and Val122Ile [5].

Treatment options for FAP focus on stabilizing or decreasing the amount of circulating amyloidogenic protein. Orthotopic liver transplantation reduces mutant TTR levels [6], with improved survival reported in patients with early-stage FAP, although deposition of wild-type TTR may continue [7-12]. Tafamidis and diflunisal stabilize 
circulating TTR tetramers, which can slow the rate of disease progression $[4,13-15]$. However, symptoms continue to worsen on treatment in many patients $[4,13-15]$, highlighting the need for new, disease-modifying treatment options for FAP.

RNA interference is a cellular process that controls gene expression, in which small interfering RNAs (siRNAs) mediate the cleavage of specific messenger RNAs (mRNAs) $[16,17]$. Lipid nanoparticles (LNPs) deliver siRNAs to hepatocytes, resulting in the robust and durable reduction in expression (so-called "knockdown") of gene targets across multiple species [18-23]. Patisiran (ALN-TTR02) comprises a TTR mRNA-specific siRNA formulated in LNPs [20]. A phase I ascending-dose study in healthy volunteers demonstrated rapid, dose-dependent, and durable knockdown of serum TTR with patisiran [20]. The objective of this study was to evaluate the safety, tolerability, pharmacokinetics (PK), and pharmacodynamics (PD) of multiple ascending intravenous (IV) doses of patisiran in patients with FAP.

\section{Methods \\ Patients}

Eligible patients were adults ( $\geq 18$ years) with biopsyproven ATTR amyloidosis and mild-to-moderate neuropathy; Karnofsky performance status $\geq 60 \%$; body mass index $17-33 \mathrm{~kg} / \mathrm{m}^{2}$; adequate liver and renal function (aspartate transaminase [AST] and alanine transaminase $[\mathrm{ALT}] \leq 2.5 \times$ the upper limit of normal [ULN], total bilirubin within normal limits, albumin $>3 \mathrm{~g} / \mathrm{dL}$, international normalized ratio $\leq 1.2$, serum creatinine $\leq 1.5$ ULN); and seronegativity for hepatitis B virus and hepatitis $C$ virus. Patients were excluded if they had a liver transplant; had surgery planned during the study; were HIV-positive; had received an investigational drug other than tafamidis or diflunisal within 30 days; had a New York Heart Association heart failure classification $>2$; were pregnant or nursing; had known or suspected systemic bacterial, viral, parasitic, or fungal infections; had unstable angina; had uncontrolled clinically significant cardiac arrhythmia; or had a prior severe reaction to a liposomal product or known hypersensitivity to oligonucleotides.

\section{Study design}

This was a multicenter, international, open-label, multipledose escalation phase II study of patisiran in patients with FAP. Cohorts of 3 patients received 2 doses of patisiran, with each dose administered as an IV infusion. Cohorts 1-3 received 2 doses of patisiran 0.01, 0.05 , and $0.15 \mathrm{mg} / \mathrm{kg}$ every 4 weeks $(\mathrm{Q} 4 \mathrm{~W})$, respectively; cohorts 4 and 5 both received 2 doses of patisiran $0.3 \mathrm{mg} / \mathrm{kg}$ Q4W. All patients in cohorts 6-9 received 2 doses of patisiran $0.3 \mathrm{mg} / \mathrm{kg}$ administered every 3 weeks
(Q3W). As lipid-based delivery systems have previously been associated with adverse immune events [24, 25], all patients received premedication prior to each patisiran infusion consisting of dexamethasone, paracetamol (acetaminophen), an $\mathrm{H} 2$ blocker (e.g. ranitidine or famotidine), and an $\mathrm{H} 1$ blocker (e.g. cetirizine, hydroxyzine, or fexofenadine) to reduce the risk of infusion-related reactions (further details on premedication regimen are provided in Additional file 1). Patisiran was administered IV at $3.3 \mathrm{~mL} / \mathrm{min}$ over $60 \mathrm{~min}$, or over $70 \mathrm{~min}$ using a micro-dosing regimen $(1.1 \mathrm{~mL} / \mathrm{min}$ for $15 \mathrm{~min}$ followed by $3.3 \mathrm{~mL} / \mathrm{min}$ for the remainder of the dose). Cumulative safety and tolerability data on all patients was reviewed by the Safety Review Committee (SRC). This study provides Class III evidence on the efficacy and safety of patisiran in patients with FAP.

\section{Standard protocol approvals, registrations, and patient consents}

The study procedures (Clinicaltrials.gov identifier: NCT01617967) were approved by the ethics committee on human experimentation at each site. All patients provided written informed consent.

\section{Outcome measures}

The primary study objective was to evaluate the safety and tolerability of multiple ascending doses of patisiran. Secondary objectives were to characterize the plasma and urine PK of patisiran, and to assess preliminary evidence of the PD effect of patisiran on serum total TTR protein levels.

Serum levels of total TTR protein were assessed for all patients using an enzyme-linked immunosorbent assay (ELISA). Additionally, wild-type and mutant TTR protein were separately and specifically measured in serum for patients with the Val30Met mutation using a proprietary mass spectrometry method (Charles River Laboratories, Quebec, Canada). Serum samples were collected at screening, and on Days 0, 1, 2, 7, 10, 14, 21, 22, 23 (Q3W only); 28, 29 (Q4W only); 30 (Q4W only); 31 (Q3W only); 35, 38 (Q4W only); and 42, 49, 56, 112, and 208 of follow-up.

Plasma concentration-time profiles were created for TTR siRNA, based on blood samples collected on Day 0 and at the following time points: pre-dose (within $1 \mathrm{~h}$ of planned dosing start), at end of infusion (EOI), at 5,10 , and $30 \mathrm{~min}$, and at 1, 2, 4, 6, 24, 48, 168, 336, 504 (Day 21, Q3W regimen only), and $672 \mathrm{~h}$ (Day 28, Q4W regimen only) post-infusion. Additional samples were collected on Days 84 and 180 for the Q4W regimens, and on Days 35, 91, and 187 for the Q3W regimen. For cohorts 3-9, blood samples on Day 0 at EOI and $2 \mathrm{~h}$ post-infusion were also analyzed for both free and 
encapsulated TTR siRNA. Serum TTR siRNA was analyzed using a validated ATTO-Probe high-performance liquid chromatography (HPLC) assay (Tandem Laboratories, Salt Lake City, Utah, USA). PK analyses were conducted using non-compartmental and/or compartmental evaluation of TTR siRNA plasma concentrationtime data to determine PK parameter estimates using the validated software program WinNonlin ${ }^{\circ}$. Urine samples were analyzed for levels of excreted TTR siRNA, and renal clearance was measured after dosing.

Serum levels of vitamin A and retinol binding protein (RBP) were measured by HPLC and nephelometry, respectively, at the same time points specified for total TTR (Biomins Specialized Medical Pathology, Lyon, France).

Safety evaluations included assessment of adverse events (AEs), electrocardiograms (ECGs), arterial oxygen saturation using pulse oximetry, vital signs, clinical laboratory safety tests, and physical examinations. AEs were defined as mild (easily tolerated with no disruption of normal daily activity), moderate (sufficient discomfort to interfere with daily activity), or severe (those which incapacitated and prevented usual activity). The numbers and percentages of patients with any treatmentemergent AE (TEAE), with any serious TEAE, with any TEAE leading to discontinuation of study medication, or with any TEAE considered a dose-limiting toxicity were summarized by dose cohort and overall. Dose-limiting toxicities included any of the following: any lifethreatening toxicity; ALT and AST $\geq 5 \times$ ULN or total bilirubin $>2.0 \mathrm{mg} / \mathrm{dL}$; an infusion reaction that required hospitalization; and any other toxicity that in the opinion of the SRC precluded administration of a second dose.

\section{Statistical analyses}

Based on the planned dose escalation scheme, we expected to enroll 27 patients. Patient populations included intent-to-treat (ITT, all patients who were enrolled and received study treatment) and per-protocol (PP, ITT patients with no major protocol violations). Safety measures were assessed in the ITT population. No substitutions were made to allow for missing data points.

Means and variances for TTR knockdown from baseline were calculated for the PP population, with baseline defined as the average of all pre-dose values. We used analysis of variance (ANOVA) and analysis of covariance (ANCOVA) to analyze the PD data (natural log transformed TTR relative to baseline), with Tukey's post hoc tests of individual pairwise comparisons (between dose levels). Nadir TTR levels were defined as the minimum level per patient during the 28-day period (21-day period for Q3W group) after each dose administration (first- dose, second-dose periods: Days $1-28,29-56$, and Days 1-21, 22-42 for Q4W and Q3W groups, respectively). Relationships between TTR and RBP or vitamin A, relative to baseline, and the relationship between wild-type and V30M TTR levels, were explored via linear regression. We formally evaluated the dose proportionality of the patisiran component in PK parameters using a power model analysis. AEs were coded using the Medical Dictionary for Regulatory Activities coding system, version 15.0, and descriptive statistics provided for AEs, laboratory data, vital signs data, and ECG interval data. All statistical analyses were performed using SAS (version 9.3 or higher) and/or R (version 2.6 or higher).

\section{Results}

Baseline demographics and disease characteristics

A total of 29 patients were enrolled across 7 countries: Brazil, France, Germany, Portugal, Spain, Sweden, and the USA. All patients were white, $69 \%$ were male, and the mean (standard deviation [SD]) age was 56 (15.6) years (Table 1$)$. The majority of patients $(76 \%)$ had the Val30Met TTR mutation. A small proportion (14\%) of patients had walking difficulties requiring the use of a stick or crutch, with the remainder having unimpaired ambulation. The majority of patients were taking a concurrent TTR tetramer stabilizer, including $48 \%$ on tafamidis and $24 \%$ using diflunisal.

\section{Patient disposition}

Of the 29 patients enrolled, all received study treatment and were included in the ITT population, and $26 \mathrm{pa}$ tients completed the study. Two patients discontinued from the study after receiving only 1 dose of patisiran: 1 patient in the $0.01 \mathrm{mg} / \mathrm{kg}$ dosing group (cohort 1) discontinued following a protocol amendment (Additional file 1), and 1 patient in the $0.3 \mathrm{mg} / \mathrm{kg}$ Q3W group withdrew from the study because of an AE. Following the protocol-related patient discontinuation in the $0.01 \mathrm{mg} / \mathrm{kg}$ Q4W group, an additional patient was enrolled and included in this dose cohort. In addition to the 2 patients who discontinued after the first dose of patisiran, 1 patient in the $0.3 \mathrm{mg} / \mathrm{kg}$ Q $4 \mathrm{~W}$ group did not complete the study due to a protocol violation (missed follow-up assessment). This patient was excluded from PD analyses after Day 28 owing to an $\mathrm{AE}$ (extravasation of the second dose of patisiran), and an additional patient was recruited to the study.

Seven patients received patisiran $0.3 \mathrm{mg} / \mathrm{kg}$ Q4W (cohorts 4 and 5) and 4 patients received patisiran $0.01 \mathrm{mg} / \mathrm{kg}$; all other study cohorts included 3 patients as described. Of the cohorts treated with patisiran $0.3 \mathrm{mg} / \mathrm{kg}, 10$ patients received their infusions over 60 min, and 9 patients (all in the Q3W group) received the 70 -min micro-dosing regimen. One patient in the 
Table 1 Baseline demographics and disease characteristics (intent-to-treat population)

\begin{tabular}{|c|c|c|c|c|c|c|}
\hline Parameter & $\begin{array}{l}0.01 \mathrm{mg} / \mathrm{kg} \\
\text { Q4W } \\
(n=4)\end{array}$ & $\begin{array}{l}0.05 \mathrm{mg} / \mathrm{kg} \\
\mathrm{Q} 4 \mathrm{~W} \\
(n=3)\end{array}$ & $\begin{array}{l}0.15 \mathrm{mg} / \mathrm{kg} \\
\mathrm{Q} 4 \mathrm{~W} \\
(n=3)\end{array}$ & $\begin{array}{l}0.3 \mathrm{mg} / \mathrm{kg} \\
\text { Q4W } \\
(n=7)\end{array}$ & $\begin{array}{l}0.3 \mathrm{mg} / \mathrm{kg} \\
\mathrm{Q} 3 \mathrm{~W} \\
(n=12)\end{array}$ & $\begin{array}{l}\text { All patients } \\
(n=29)\end{array}$ \\
\hline \multicolumn{7}{|l|}{ Sex, n (\%) } \\
\hline Male & $3(75.0)$ & $3(100.0)$ & $2(66.7)$ & $3(42.9)$ & $9(75.0)$ & $20(69.0)$ \\
\hline Female & $1(25.0)$ & 0 & $1(33.3)$ & $4(57.1)$ & $3(25.0)$ & $9(31.0)$ \\
\hline \multicolumn{7}{|l|}{ Age, years } \\
\hline Mean (SD) & $65.8(8.96)$ & $55.7(24.83)$ & $41.7(2.52)$ & $58.7(16.07)$ & $53.8(15.6)$ & $55.6(15.61)$ \\
\hline \multicolumn{7}{|l|}{ Race, n (\%) } \\
\hline White/Caucasian & $4(100.0)$ & $3(100.0)$ & $3(100.0)$ & $7(100.0)$ & $12(100.0)$ & $29(100.0)$ \\
\hline \multicolumn{7}{|l|}{ Country } \\
\hline Portugal & 0 & $1(33.3)$ & $3(100.0)$ & $2(28.6)$ & $3(25.0)$ & $9(31.0)$ \\
\hline Sweden & $2(50.0)$ & $1(33.3)$ & 0 & $2(28.6)$ & $1(8.33)$ & $6(20.7)$ \\
\hline France & $2(50.0)$ & $1(33.3)$ & 0 & $2(28.6)$ & $3(25.0)$ & $8(27.6)$ \\
\hline Brazil & 0 & 0 & 0 & 0 & $1(8.33)$ & $1(3.4)$ \\
\hline Germany & 0 & 0 & 0 & 0 & $1(8.33)$ & $1(3.4)$ \\
\hline Spain & 0 & 0 & 0 & 0 & $3(25.0)$ & $3(10.3)$ \\
\hline United States & 0 & 0 & 0 & $1(14.3)$ & 0 & $1(3.4)$ \\
\hline \multicolumn{7}{|l|}{ TTR genotype, n (\%) } \\
\hline Val30Met & $2(50.0)$ & $2(66.7)$ & $3(100.0)$ & $6(85.7)$ & $9(75.0)$ & $22(75.9)$ \\
\hline Other $^{a}$ & $2(50.0)$ & $1(33.3)$ & 0 & $1(14.3)$ & $3(25.0)$ & $7(24.1)$ \\
\hline Mean (SD) serum TTR at baseline, $\mu \mathrm{g} / \mathrm{mL}$ & $272.9(98.96)$ & $226.5(12.67)$ & $276.1(7.65)$ & $242.6(38.30)$ & $235.5(44.45)$ & $245.64(49.44)$ \\
\hline \multicolumn{7}{|l|}{ FAP stage ${ }^{b}$} \\
\hline 1 & - & - & - & - & - & $25(86.2)$ \\
\hline 2 & - & - & - & - & - & $4(13.8)$ \\
\hline \multicolumn{7}{|l|}{ Prior exposure to ALN-TTR01 ${ }^{c}$} \\
\hline Yes & $2(50.0)$ & 0 & $2(66.7)$ & $3(42.9)$ & $2(16.67)$ & $9(31.0)$ \\
\hline No & $2(50.0)$ & $3(100.0)$ & $1(33.3)$ & $4(57.1)$ & $10(83.33)$ & $20(69.0)$ \\
\hline \multicolumn{7}{|l|}{ Concurrent TTR stabilizer use } \\
\hline Diflunisal & $2(50.0)$ & $1(33.3)$ & 0 & $3(42.9)$ & $1(33.3)$ & $7(24.1)$ \\
\hline Tafamidis & 0 & $1(33.3)$ & $2(66.7)$ & $4(57.1)$ & $7(66.7)$ & $14(48.3)$ \\
\hline
\end{tabular}

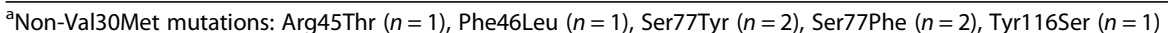

${ }^{b}$ FAP stage: $1=$ unimpaired ambulation, mostly mild neuropathy in lower limbs; $2=$ assistance with ambulation required, mostly moderate neuropathy with progression to lower limbs, upper limbs, and trunk; 3 = wheelchair-bound or bedridden, severe neuropathy of all limbs

${ }^{C}$ ALN-TTR01 was the first-generation siRNA-LNP used in phase I studies in patients with ATTR

ATTR TTR amyloid fibrils, FAP familial amyloid polyneuropathy; Q3W every 3 weeks; Q4W every 4 weeks; SD standard deviation; siRNA-LNP small interfering RNA-lipid nanoparticles; $T R$ transthyretin

$0.05 \mathrm{mg} / \mathrm{kg}$ Q4W cohort 2 did not meet the eligibility criteria because of liver function data, but was given a waiver to enter the study.

\section{Efficacy and PD}

Mean (SD) baseline serum TTR protein levels were similar across the dose cohorts: 272.9 (98.86), 226.5 (12.67), 276.1 (7.65), 242.6 (38.30), and $235.5(44.45) \mu \mathrm{g} / \mathrm{mL}$ for the $0.01,0.05,0.15,0.3 \mathrm{Q} 4 \mathrm{~W}$, and $0.3 \mathrm{mg} / \mathrm{kg}$ Q3W dosage groups, respectively.
In comparison to the $0.01 \mathrm{mg} / \mathrm{kg}$ dose cohort, a significant reduction in nadir TTR levels $(p<0.001$ by post hoc tests after ANCOVA) was observed after the first and second doses of patisiran in the $0.3 \mathrm{mg} / \mathrm{kg}$ Q4W and Q3W cohorts (Fig. 1 and Table 2). In patients given $0.3 \mathrm{mg} / \mathrm{kg}$ Q3W, mean \pm SD TTR knockdown from baseline at nadir was $83.8 \pm 5.1 \%$ and $86.7 \pm 7.0 \%$ after the first and second doses, respectively, with maximum knockdown of $96 \%$. In patients given the same dose Q4W, mean \pm SD TTR knockdown from baseline at nadir was $82.9 \pm 5.4 \%$ and 


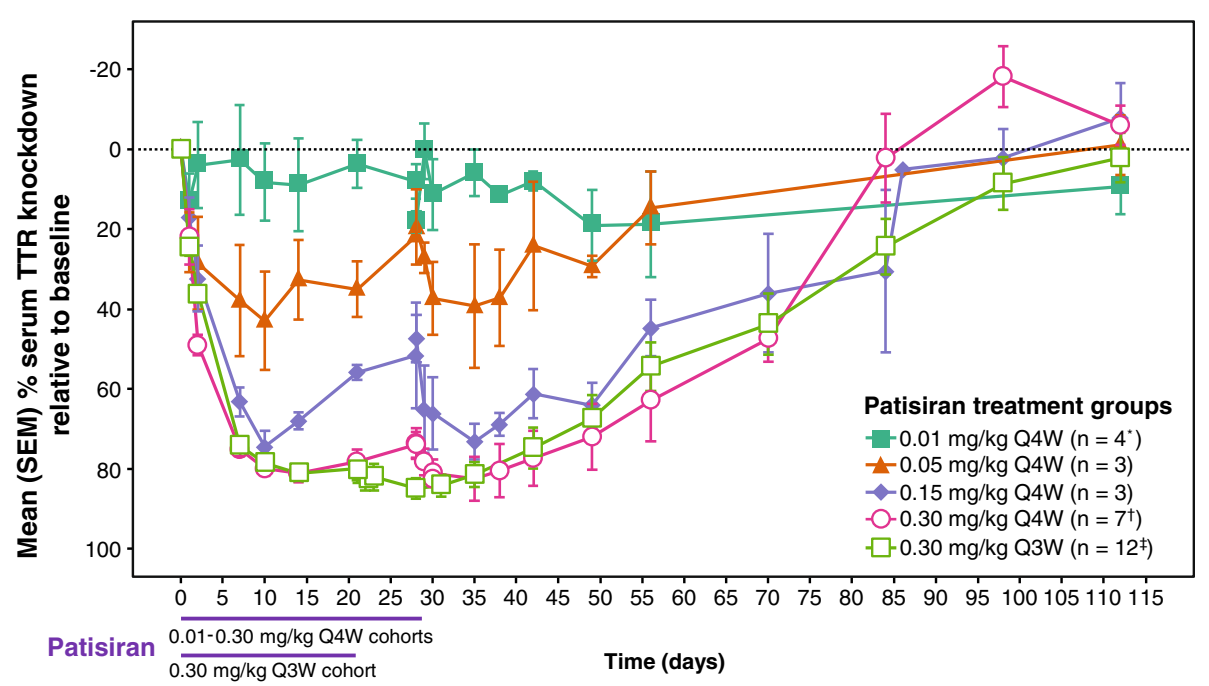

Fig. 1 Dose response and duration of TTR knockdown. Mean ( \pm SEM) percentage of baseline serum concentration-time profile. Q3W: every 3 weeks; Q4W: every 4 weeks; SEM: standard error of the mean; TTR: transthyretin. *Includes first dose data from additional patient prior to protocol amendment. ${ }^{\dagger}$ Excludes post-Day 28 data from patient who experienced drug extravasation during second infusion. ${ }^{\ddagger}$ One patient discontinued before the second dose of patisiran

$85.7 \pm 9.6 \%$ after the first and second doses, respectively, with maximum knockdown of $90.8 \%$. Mean TTR knockdown from baseline of $\geq 80 \%$ was maintained between doses in the Q3W cohort, yet TTR levels recovered to < $80 \%$ between doses with the Q4W regimen. In patients with the Val30Met mutation, a very similar degree of knockdown was observed for wild-type and mutant TTR (Fig. 2a and b). The levels of mutant and wild-type TTR knockdown were not assessed for each of the rarer non-Val30Met genotypes, although overall knockdown, as measured by ELISA, was indistinguishable between patients with Val30Met or non-Val30Met mutations. The level of serum TTR knockdown was highly correlated with the reduction in circulating level of RBP $\left(\mathrm{r}^{2}=0.89, p<10^{-15}\right)$ and vitamin $\mathrm{A}\left(\mathrm{r}^{2}=0.89, p<10^{-15}\right)$ (Additional file 2: Figure S1).
Although patients taking tafamidis or diflunisal had significantly increased baseline levels of serum TTR compared with patients not taking stabilizer therapy $(p<0.001$ by ANOVA) (Additional file 3: Figure S2a), patisiran administration resulted in a similar degree of TTR knockdown in these 2 patient groups (Additional file 3: Figure S2b).

\section{PK}

Mean concentrations of the patisiran TTR siRNA component decreased after EOI (Additional file 4: Figure S3), and there was no accumulation of siRNA following the second dose on Day 21/28. Measurements of encapsulated versus un-encapsulated concentrations of TTR siRNA after each dose indicated stability of the circulating LNP formulation. For both the first and second doses, the

Table 2 Serum TTR knockdown by dose group

\begin{tabular}{|c|c|c|c|c|}
\hline \multirow[t]{2}{*}{ Dose group (mg/kg) } & \multicolumn{2}{|l|}{ Dose 1} & \multicolumn{2}{|l|}{ Dose 2} \\
\hline & Maximum TTR KD (\%) & TTR KD at Nadir (Mean $\% \pm$ SD) & Maximum TTR KD (\%) & TTR KD at Nadir (Mean $\% \pm$ SD) \\
\hline $0.01 \mathrm{Q} 4 \mathrm{~W}\left(n=4^{\mathrm{a}}\right)$ & 37.8 & $22.1 \pm 12.5$ & 34.4 & $32.9 \pm 2.3$ \\
\hline $0.05 \mathrm{Q} 4 \mathrm{~W}(n=3)$ & 58.0 & $48.4 \pm 16.2$ & 58.5 & $46.9 \pm 15.0$ \\
\hline $0.15 \mathrm{Q} 4 \mathrm{~W}(n=3)$ & 81.7 & $74.5 \pm 6.8^{* * *}$ & 86.0 & $77.0 \pm 7.8$ \\
\hline $0.3 \mathrm{Q} 4 \mathrm{~W}\left(n=7^{\mathrm{b}}\right)$ & 87.5 & $82.9 \pm 5.4^{* * *}$ & 90.8 & $85.7 \pm 9.6^{* * *}$ \\
\hline $0.3 \mathrm{Q} 3 \mathrm{~W}\left(n=12^{\mathrm{C}}\right)$ & 94.2 & $83.8 \pm 5.1^{* * *}$ & 96.0 & $86.7 \pm 7.0^{* * *}$ \\
\hline
\end{tabular}

p values from ANCOVA models including baseline TTR as covariate and dose groups as factor; models significant at $p<0.001$ for Dose $1, p<0.001$ for Dose 2 *** $p<0.001$ vs. $0.01 \mathrm{mg} / \mathrm{kg}$ group

ancludes first-dose data from additional patient prior to protocol amendment

${ }^{b}$ Excludes post-day 28 data from patient who experienced drug extravasation during second infusion

${ }^{\mathrm{C}}$ One patient discontinued the study before second dose of patisiran

ANCOVA analysis of variance; KD knockdown; Q3W every 3 weeks; $Q 4 W$ every 4 weeks; SD standard deviation; $T R R$ transthyretin 

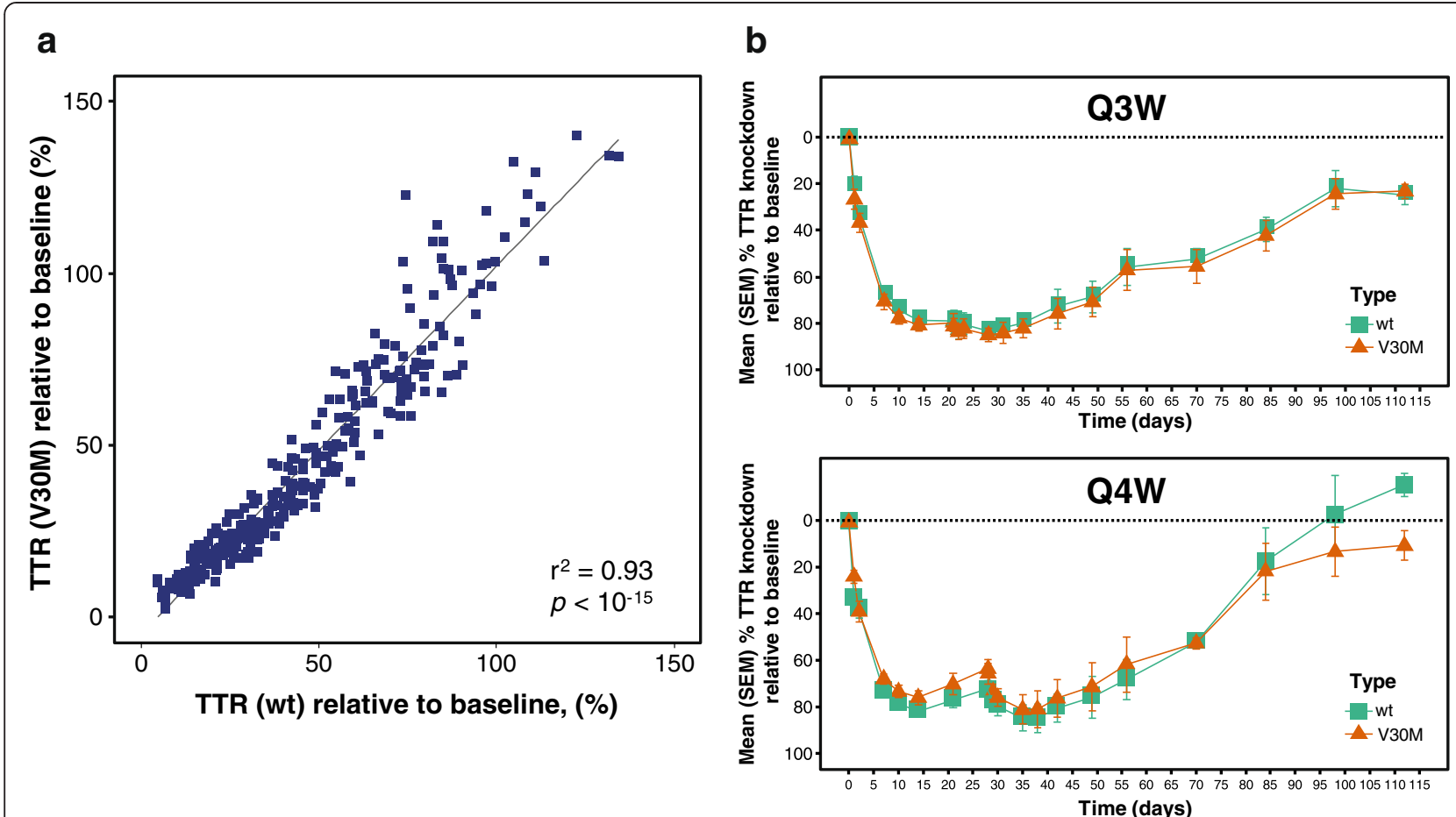

Fig. 2 Effect of patisiran on wild-type and mutant TTR in patients with the Val30Met mutation. a All post-dose data. b Patisiran $300 \mathrm{mg} / \mathrm{kg}$ groups (error bars represent SEM). Q3W: every 3 weeks; Q4W: every 4 weeks; SEM: standard error of the mean; TTR: transthyretin; wt: wild-type

mean values for maximum plasma concentration $\left(\mathrm{C}_{\max }\right)$ and area under the plasma concentration-time curve from zero to the last measurable time point $\left(\mathrm{AUC}_{0-\text { last }}\right)$ increased in a dose-proportional manner over the dose range tested. $\mathrm{C}_{\max }$ and $\mathrm{AUC}_{0-\text { last }}$ after doses 1 and 2 were comparable, with no accumulation. The median terminal half-life of patisiran at Day 0 and Days $21 / 28$ was $39-59 \mathrm{~h}$ at doses $>0.01 \mathrm{mg} / \mathrm{kg}$, and was relatively unchanged when comparing doses 1 and 2 for each dose cohort.

\section{Safety and tolerability}

The most common TEAE related to study drug was mild-to-moderate infusion-related reaction (IRR), which occurred in 3/29 patients overall (10.3\%), all in the $0.3 \mathrm{mg} / \mathrm{kg}$ Q4W group (Table 3); none of these TEAEs led to discontinuation of treatment. IRR-associated symptoms were tachycardia, decreased oxygen saturation, dizziness, abdominal pain, bronchospasm, dyspnea, erythema, chills, pallor, pyrexia, and tachypnea. For 1 patient with a mild reaction, the event was managed by prolonging the IV infusion time. Of note, no IRRs were reported in the patient cohort that received $0.3 \mathrm{mg} / \mathrm{kg} \mathrm{Q} 3 \mathrm{~W}(\mathrm{n}=12), 9$ of whom received their infusion with the micro-dosing regimen administered over 70 min. Aside from IRRs, no drug-related TEAE was observed in more than 1 patient per dosage group across the study.
There were no dose-limiting toxicities or deaths due to TEAEs reported during the course of the study. The majority of TEAEs were of mild or moderate intensity. Four serious AEs (SAEs) were reported in 1 patient in the $0.3 \mathrm{mg} / \mathrm{kg}$ Q3W group (urinary tract infection, sepsis, nausea, and vomiting), and the patient withdrew from the study because of the nausea and vomiting. An additional SAE (extravasation-related cellulitis) was recorded in 1 patient in the $0.3 \mathrm{mg} / \mathrm{kg}$ Q4W group. The SAEs of nausea and vomiting, and cellulitis were each recorded by the Investigator as related to the study drug.

No clinically significant changes in liver function tests, renal function, or hematologic parameters were recorded. Transient increases in white blood cell count were observed approximately $24 \mathrm{~h}$ after each patisiran infusion, which were considered related to dexamethasone premedication. There were no substantial changes in serum G-CSF, IFN- $\alpha$, IFN- $\gamma$, IL-1b, IL-12, and TNF- $\alpha$ cytokines. Values were below the lower limit of detection for most patients at the majority of time points. Transient increases in mean levels of IL-6, IL-1RA, and IP10 were observed after patisiran infusion in the $0.3 \mathrm{mg} / \mathrm{kg}$ Q4W group (and in the $0.3 \mathrm{mg} / \mathrm{kg}$ Q3W group for IL-6), although levels returned to baseline within $24 \mathrm{~h}$. Transient increases in mean levels of complement factor $\mathrm{Bb}$ were also seen after infusion of patisiran doses 0.05$0.3 \mathrm{mg} / \mathrm{kg}$. None of these elevations were associated with AEs. 
Table 3 Treatment-emergent adverse events related or possibly related to patisiran (ITT [safety] population)

\begin{tabular}{|c|c|c|c|c|c|c|}
\hline \multirow[t]{4}{*}{ Preferred term, n (\%) } & \multicolumn{6}{|l|}{ Patisiran } \\
\hline & $0.01 \mathrm{mg} / \mathrm{kg}$ & $0.05 \mathrm{mg} / \mathrm{kg}$ & $0.15 \mathrm{mg} / \mathrm{kg}$ & $0.3 \mathrm{mg} / \mathrm{kg}$ & $0.3 \mathrm{mg} / \mathrm{kg}$ & \\
\hline & Q4W & Q4W & Q4W & Q4W & Q3W & Overall \\
\hline & $(n=4)$ & $(n=3)$ & $(n=3)$ & $(n=7)$ & $(n=12)$ & $(n=29)$ \\
\hline Infusion-related reaction & 0 & 0 & 0 & $3(42.9)$ & 0 & $3(10.3)$ \\
\hline Back pain & 0 & 0 & 0 & $2(28.6)$ & 0 & $2(6.9)$ \\
\hline Asthenia & 0 & 0 & 0 & $1(14.3)$ & $1(8.3)$ & $2(6.9)$ \\
\hline Leukocytosis & 0 & 0 & 0 & 0 & $1(8.3)$ & $1(3.4)$ \\
\hline Neutrophilia & 0 & 0 & 0 & 0 & $1(8.3)$ & $1(3.4)$ \\
\hline Cellulitis $^{\mathrm{a}}$ & 0 & 0 & 0 & $1(14.3)$ & 0 & $1(3.4)$ \\
\hline Lymphangitis & 0 & 0 & 0 & $1(14.3)$ & 0 & $1(3.4)$ \\
\hline Polyuria & 0 & 0 & 0 & $1(14.3)$ & 0 & $1(3.4)$ \\
\hline Nausea/vomiting & 0 & 0 & 0 & 0 & $1(8.3)$ & $1(3.4)$ \\
\hline Facial erythema & 0 & 0 & 0 & 0 & $1(8.3)$ & $1(3.4)$ \\
\hline Dry mouth & 0 & 0 & 0 & 0 & $1(8.3)$ & $1(3.4)$ \\
\hline Pyrexia & 0 & 0 & 0 & 0 & $1(8.3)$ & $1(3.4)$ \\
\hline Dysphagia & 0 & 0 & 0 & 0 & $1(8.3)$ & $1(3.4)$ \\
\hline
\end{tabular}

${ }^{\mathrm{a}}$ Due to drug extravasation

ITT intent-to-treat; Q3W every 3 weeks; Q4W every 4 weeks

\section{Discussion}

These phase II data demonstrate that administration of patisiran to patients with FAP led to robust, dosedependent, and statistically significant knockdown of serum TTR protein levels. Mean sustained reduction in TTR of $>80 \%$ was achieved with 2 consecutive doses of patisiran $0.3 \mathrm{mg} / \mathrm{kg}$ dosed every $3-4$ weeks, with a maximum knockdown of $96 \%$ achieved in the Q3W group. These knockdown rates are consistent with the rates observed in the single ascending dose, placebo-controlled phase I study of patisiran [20]. Evidence from other systemic amyloidotic diseases indicates that as little as $50 \%$ reduction of the disease-causing protein can result in clinical disease improvement or stabilization [26, 27], illustrating the potential of this therapy. The degree of TTR knockdown with patisiran was not affected by patients taking tafamidis or diflunisal, suggesting that these TTR stabilizer drugs do not interfere with the pharmacologic activity of patisiran. In patients with the Val30Met mutation, patisiran suppressed production of both mutated and wild-type TTR; the latter remains amyloidogenic in patients with late-onset FAP after liver transplantation [28, 29].

Multiple doses of patisiran were shown to be generally safe and well tolerated in this study. The majority of AEs were mild or moderate in severity and no dose-limiting toxicities were observed. While IRRs were the most common drug-related TEAE seen at $0.3 \mathrm{mg} / \mathrm{kg}$, no IRRs were reported in the $0.3 \mathrm{mg} / \mathrm{kg}$ group that received the micro-dosing infusion regimen over $70 \mathrm{~min}$. Premedication was used to reduce the risk of IRRs, and is thus considered in assessment of the tolerability of this regimen. Glucocorticoids such as dexamethasone can increase white blood cell levels [30], as observed transiently in this study, and TEAEs potentially related to premedication will be monitored during longer term patisiran administration. The generally favorable tolerability profile observed in this study, with most patients receiving TTR stabilizers, is encouraging with respect to the potential concurrent use of these agents with patisiran.

This multi-dose study defined patisiran $0.3 \mathrm{mg} / \mathrm{kg}$ Q3W delivered using the 70-min micro-dosing regimen as the formulation and dosage to administer in the ongoing open-label extension (OLE) phase of patients with FAP. Whilst both Q3W and Q4W achieved potent TTR knockdown, the level of TTR suppression was better maintained between doses with the Q3W schedule, supporting its future investigation. Preliminary data from the OLE study show sustained TTR knockdown of $\sim 80 \%$ after 168 days (9 doses), with no reported SAEs $(n=27)$ [31]. The OLE study preliminary safety data are consistent with the tolerability of patisiran observed in this trial, with TEAEs all mild or moderate in severity [31].

Patient baseline demographics and disease characteristics in the present study were similar to those reported for other recent FAP studies. In particular, the mean age (55.6 years) was comparable to that reported for a large natural history study of patients with FAP (56.4 years; $\mathrm{n}=283$ ) [32] and for a phase II/III diflunisal study (59.7 years; $n=130$ ) [15], but was greater than the 39.8 / 
38.4 years reported for the tafamidis/placebo arms in the tafamidis phase II/III study $(n=125)$ [14]. Some $75.9 \%$ of patients in the present study had the Val30Met mutation compared with $58.5 \%$ (161/275 evaluable patients), $54.6 \%$, and $100 \%$ in the natural history, diflunisal, and tafamidis studies, respectively $[14,15,32]$.

The results of this study supported the initiation of a phase III study of patisiran. APOLLO is a randomized, placebo-controlled, phase III study of patisiran $0.3 \mathrm{mg} / \mathrm{kg}$ Q3W administered using the 70-min microdosing schedule in recruiting patients with Val30Met or non-Val30Met FAP who are not taking TTR stabilizers. The primary study endpoint is disease progression, measured as the change from baseline in $\mathrm{mNIS}+7$ score at 18 months (Clinical trial identifier NCT01960348). Secondary endpoints include measures of quality of life and disease burden, changes from baseline in motor and autonomic neuropathy measures, and safety.

\section{Conclusion}

In conclusion, the results of this phase II study provide evidence that the investigational agent patisiran was generally well tolerated and effective in reducing both mutant and wild-type TTR levels in patients with FAP. The $0.3 \mathrm{mg} / \mathrm{kg}$ Q3W dosing schedule is under investigation in the phase III APOLLO trial.

\section{Additional files}

Additional file 1: Supplemental materials. (DOCX $36 \mathrm{~kb}$ )

Additional file 2: Figure S1. Correlation of serum TTR knockdown with change from baseline in serum vitamin A and RBP. RBP: retinol binding protein; TTR: transthyretin. (DOCX 235 kb)

Additional file 3: Figure S2. TTR knockdown with patisiran in patients taking a tetramer stabilizer. (a) Baseline TTR levels by stabilizer use (all cohorts). (b) TTR knockdown by patisiran $(0.3 \mathrm{mg} / \mathrm{kg}$ cohorts; error bars represent SEM). SEM: standard error of the mean; TTR: transthyretin. (DOCX $260 \mathrm{~kb}$ )

Additional file 4: Figure S3. Mean $( \pm S D)$ plasma concentration-time profiles of patisiran following $1 \mathrm{~h}$ intravenous infusion (semi-log scale) for: (a) First (Day 0) and (b) second (Day 21/28) doses. Q3W: every 3 weeks; Q4W: every 4 weeks; SD: standard deviation. (DOCX 196 kb)

\footnotetext{
Abbreviations

AE: Adverse event; ALT: Alanine transaminase; ANCOVA: Analysis of covariance; ANOVA: Analysis of variance; AST: Aspartate transaminase; ATTR: TTR amyloid fibrils; $A \cup C_{0-\text { last }}$ : Area under the plasma concentrationtime curve from zero to the last measurable time point; $C_{\text {max }}$ : Maximum plasma concentration; ECG: Electrocardiograms; EOI: End of infusion; FAP: Familial amyloid polyneuropathy; HPLC: High-performance liquid chromatography; IRR: Infusion-related reaction; ITT: Intent-to-treat; IV: Intravenous; KD: Knockdown; LNP: Lipid nanoparticles; OLE: Open-label extension; PD: Pharmacodynamics; PK: Pharmacokinetics; PP: Per-protocol; Q3W: Every 3 weeks; Q4W: Every 4 weeks; RBP: Retinol binding protein; SAE: Serious AE; SD: Standard deviation; siRNA: Small interfering RNA; siRNALNP: Small interfering RNA-lipid nanoparticle; SRC: Safety Review Committee; TEAE: Treatment-emergent AE; TTR: Transthyretin; ULN: Upper limit of normal.
}

\section{Competing interests}

OS's department has received honoraria from Pfizer for his participation as a speaker at conferences and meetings, and he is currently participating in clinical studies conducted by Pfizer and Alnylam Pharmaceuticals. TC's hospital was paid per protocol for clinical trials from FoldRx, Pfizer, ISIS, and Alnylam Pharmaceuticals. She also received financial support from Pfizer and Alnylam Pharmaceuticals to attend scientific meetings. She is also involved with the speakers' bureau of Pfizer for which she has received honoraria. J Buades is a consultant and investigator for Alnylam Pharmaceuticals. JP is an investigator for Alnylam Pharmaceuticals. IC received financial support from Pfizer as a consultant and for conferences and symposia, from Alnylam Pharmaceuticals as a primary investigator, and from ISIS Pharmaceuticals as a primary investigator. She also serves on the THAOS scientific advisory board, which is financially supported by Pfizer. J Berk received travel expenses from Alnylam and financial support for study preparation for Alnylam and Isis Pharmaceuticals in 2014. HS reports no disclosure relevant to this manuscript. MW-C received honoraria and travel expenses from Pfizer as a consultant from 2007 to 2014, and for conferences and symposia; honoraria, and travel expenses from Alnylam Pharmaceuticals as a consultant from 2011 to 2014, and for conferences; and travel expenses from Isis Pharmaceuticals in 2014. JMC reports no disclosure relevant to this manuscript. BRB, AV, and JG are employees and shareholders of Alnylam Pharmaceuticals Inc. DA received honoraria from Pfizer for conferences and symposia, from Alnylam Pharmaceuticals as a consultant between 2011 and 2012, and from Isis as a consultant between 2011 and 2012. He received financial support from Pfizer and Alnylam Pharmaceuticals to attend scientific meetings.

\section{Authors' contributions}

OS reviewing and revising the manuscript for content, study concept or design, acquisition of data, analysis, or interpretation of data. TC reviewing and revising the manuscript for content, study concept or design, acquisition of data, analysis, or interpretation of data. J Buades reviewing and revising the manuscript for content, study concept or design, acquisition of data, analysis, or interpretation of data. JP reviewing and revising the manuscript for content, study concept or design, acquisition of data, analysis, or interpretation of data. IC reviewing and revising the manuscript for content, study concept or design, acquisition of data, analysis, or interpretation of data. J Berk reviewing and revising the manuscript for content, study concept or design, acquisition of data, analysis, or interpretation of data. HS reviewing and revising the manuscript for content, study concept or design, acquisition of data, analysis, or interpretation of data. MW-C reviewing and revising the manuscript for content, study concept or design, acquisition of data, analysis, or interpretation of data. JMC reviewing and revising the manuscript for content, study concept or design, acquisition of data, analysis, or interpretation of data. BRB reviewing and revising the manuscript for content, study concept or design, acquisition of data, analysis, or interpretation of data. $\mathrm{A} V$ reviewing and revising the manuscript for content, study concept or design, acquisition of data, analysis, or interpretation of data. JG reviewing and revising the manuscript for content, study concept or design, acquisition of data, analysis, or interpretation of data. DA reviewing and revising the manuscript for content, study concept or design, acquisition of data, analysis, or interpretation of data. All authors read and approved the final manuscript.

\section{Acknowledgements}

All authors were responsible for reviewing and interpreting the data. The authors received editorial support from Adelphi Communications Ltd. Biostatistical analyses were conducted by Veristat LLC, Holliston, MA, USA. The authors acknowledge the support of the following co-investigators: Mercedes Uson, Carles Montala, and Cristina Descals (Hospital Son Llatzer, Palma de Mallorca, Spain), and Cecile Cauquil and Marie Theaudin (Univ Paris-Sud, Le Kremlin-Bicêtre, Paris, France). The authors also acknowledge contributions from Dorothee Lamann (Universitätsklinikum Münster, Münster, Germany) who provided technical support and Vanessa Benito who was the Study Coordinator at the Hospital Son Llatzer, Palma de Mallorca, Spain. This study was sponsored by Alnylam Pharmaceuticals, Inc.

\section{Author details}

${ }^{1}$ Department of Public Health and Clinical Medicine, Umeå University, 90187 Umeå, Sweden. ${ }^{2}$ Hospital de Santo António, Centro Hospitalar do Porto, 4099-001 Porto, Portugal. 'Servicio de Medicina Interna, Hospital Son Llatzer, Carretera Manacor KM, 7198 Palma de Mallorca, Spain. ${ }^{4}$ Hôpital de La 
Timone, 264 rue Saint Pierre, 13005 Marseille, France. ${ }^{5}$ Centro Hospitalar Lisboa Norte-Hospital de Santa Maria, Piso 7, Av, Prof Eqas Moniz, Lisboa

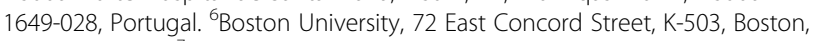
MA 02118, USA. 'Universitätsklinikum Münster, Transplant Hepatology, Domagkstr. 3A, Munster 48149, Germany. ${ }^{8}$ Hospital Universitário Clementino Fraga Filho, Federal University of Rio de Janeiro, llha do Fundao, Rio de Janeiro CEP21941-913, Brazil. ${ }^{9}$ Hospital Clinic, University of Barcelona, IDIBAPS, Escalera 12 (5 Planta), C/ Villarroel, 170, Barcelona 8036, Spain. ${ }^{10}$ Alnylam Pharmaceuticals, 300 Third Street, Cambridge, MA 02142, USA. ${ }^{11}$ National Reference Center for FAP (NNERF)/ APHP/ INSERM U 1191/ Hôpital de Bicêtre, 78, rue du General Leclerc, 94275 Le Kremlin-Bicêtre, France.

Received: 16 April 2015 Accepted: 25 August 2015 Published online: 04 September 2015

\section{References}

1. Westermark P, Sletten K, Johansson B, Cornwell GG. Fibril in senile systemic amyloidosis is derived from normal transthyretin. Proc Natl Acad Sci U S A. 1990;87(7):2843-5.

2. Coutinho P, Martins da Silva A, Lopes Lima J, Resende Barbosa A. Forty years of experience with type I amyloid neuropathy. Review of 483 cases. In: Glenner GG, Pinho e Costa P, Falcao de Freitas A, editors. Amyloid and Amyloidosis. Amsterdam: Excerpta Medica; 1980. p. 88-93.

3. Hou X, Aguilar MI, Small DH. Transthyretin and familial amyloidotic polyneuropathy. Recent progress in understanding the molecular mechanism of neurodegeneration. FEBS J. 2007;274(7):1637-50.

4. Coelho T, Maia LF, da Silva AM, Cruz MW, Planté-Bordeneuve V, Suhr OB, et al. Long-term effects of tafamidis for the treatment of transthyretin familial amyloid polyneuropathy. J Neurol. 2013;260(11):2802-14.

5. Connors LH, Prokaeva T, Lim A, Théberge R, Falk RH, Doros G, et al. Cardiac amyloidosis in African Americans: comparison of clinical and laboratory features of transthyretin V122I amyloidosis and immunoglobulin light chain amyloidosis. Am Heart J. 2009;158(4):607-14.

6. Holmgren G, Steen L, Ekstedt J, Groth CG, Ericzon BG, Eriksson S, et al. Biochemical effect of liver transplantation in two Swedish patients with familial amyloidotic polyneuropathy (FAP-met30). Clin Genet. 1991:40(3):242-6.

7. Ericzon BG, Wilczek HE, Larsson M, Wijayatunga P, Stangou A, Rodrigues Pena J, et al. Liver transplantation for hereditary transthyretin amyloidosis: after 20 years still the best therapeutic alternative? Transplantation 2015;99(9):1847-54.

8. Okamoto S, Wixner J, Obayashi K, Ando Y, Ericzon BG, Friman S, et al. Liver transplantation for familial amyloidotic polyneuropathy: impact on Swedish patients' survival. Liver Transpl. 2009;15(10):1229-35.

9. Yazaki M, Mitsuhashi S, Tokuda T, Kametani F, Takei YI, Koyama J, et al. Progressive wild-type transthyretin deposition after liver transplantation preferentially occurs onto myocardium in FAP patients. Am J Transplant. 2007;7(1):235-42.

10. Stangou AJ, Hawkins PN, Heaton ND, Rela M, Monaghan M, Nihoyannopoulos $P$, et al. Progressive cardiac amyloidosis following liver transplantation for familial amyloid polyneuropathy: implications for amyloid fibrillogenesis. Transplantation. 1998;66(2):229-33.

11. Yamashita T, Ando Y, Okamoto S, Misumi Y, Hirahara T, Ueda M, et al. Long-term survival after liver transplantation in patients with familial amyloid polyneuropathy. Neurology. 2012;78(9):637-43.

12. Adams D, Samuel D, Goulon-Goeau C, Nakazato M, Costa PM, Feray C, et al. The course and prognostic factors of familial amyloid polyneuropathy after liver transplantation. Brain. 2000;123(Pt 7):1495-504.

13. Lozeron $P$, Théaudin M, Mincheva Z, Ducot B, Lacroix C, Adams D, et al. Effect on disability and safety of Tafamidis in late onset of Met30 transthyretin familial amyloid polyneuropathy. Eur J Neurol. 2013:20(12):1539-45.

14. Coelho T, Maia LF. Martins da Silva A, Waddington CM, Planté-Bordeneuve $V$, Lozeron $P$, et al. Tafamidis for transthyretin familial amyloid polyneuropathy: a randomized, controlled trial. Neurology. 2012;79(8):785-92.

15. Berk JL, Suhr OB, Obici L, Sekijima Y, Zeldenrust SR, Yamashita T, et al. Repurposing diflunisal for familial amyloid polyneuropathy: a randomized clinical trial. JAMA. 2013;310(24):2658-67.

16. Elbashir SM, Harborth J, Lendeckel W, Yalcin A, Weber K, Tuschl T. Duplexes of 21-nucleotide RNAs mediate RNA interference in cultured mammalian cells. Nature. 2001;411(6836):494-8.
17. Fire A, Xu S, Montgomery MK, Kostas SA, Driver SE, Mello CC. Potent and specific genetic interference by double-stranded RNA in Caenorhabditis elegans. Nature. 1998;391(6669):806-11.

18. Akinc A, Querbes W, De S, Qin J, Frank-Kamenetsky M, Jayaprakash KN, et al. Targeted delivery of RNAi therapeutics with endogenous and exogenous ligand-based mechanisms. Mol Ther. 2010;18(7):1357-64.

19. Akinc A, Bettencourt BR, Maier MA. Development of RNAi therapeutics. In: Smyth Templeton N, editor. Gene and Cell Therapy: Therapeutic Mechanisms and Strategies: CRC Press, Taylor \& Francis Group: Boca Raton, USA; 2015. p. 493-519.

20. Coelho T, Adams D, Silva A, Lozeron P, Hawkins PN, Mant T, et al. Safety and efficacy of RNAi therapy for transthyretin amyloidosis. N Engl J Med. 2013;369(9):819-29.

21. Fitzgerald K, Frank-Kamenetsky M, Shulga-Morskaya S, Liebow A, Bettencourt BR, Sutherland JE, et al. Effect of an RNA interference drug on the synthesis of proprotein convertase subtilisin/kexin type 9 (PCSK9) and the concentration of serum LDL cholesterol in healthy volunteers: a randomised, single-blind, placebo-controlled, phase 1 trial. Lancet. 2014;383(9911):60-8.

22. Frank-Kamenetsky M, Grefhorst A, Anderson NN, Racie TS, Bramlage B, Akinc A, et al. Therapeutic RNAi targeting PCSK9 acutely lowers plasma cholesterol in rodents and LDL cholesterol in nonhuman primates. Proc Natl Acad Sci U S A. 2008;105(33):11915-20.

23. Zimmermann TS, Lee AC, Akinc A, Bramlage B, Bumcrot D, Fedoruk MN, et al. RNAi-mediated gene silencing in non-human primates. Nature. 2006:441(7089):111-4.

24. Szebeni J, Muggia F, Gabizon A, Barenholz Y. Activation of complement by therapeutic liposomes and other lipid excipient-based therapeutic products: prediction and prevention. Adv Drug Deliv Rev. 2011;63(12):1020-30.

25. Rowinsky EK, Donehower RC. Paclitaxel (taxol). N Engl J Med. 1995;332(15):1004-14.

26. Lachmann HJ, Goodman HJ, Gilbertson JA, Gallimore JR, Sabin CA, Gillmore $\mathrm{JD}$, et al. Natural history and outcome in systemic AA amyloidosis. N Engl J Med. 2007;356(23):2361-71.

27. Lachmann HJ, Gallimore R, Gillmore JD, Carr-Smith HD, Bradwell AR, Pepys $M B$, et al. Outcome in systemic AL amyloidosis in relation to changes in concentration of circulating free immunoglobulin light chains following chemotherapy. Br J Haematol. 2003;122(1):78-84.

28. Yazaki M, Liepnieks JJ, Kincaid JC, Benson MD. Contribution of wild-type transthyretin to hereditary peripheral nerve amyloid. Muscle Nerve. 2003;28(4):438-42.

29. Liepnieks JJ, Zhang LQ, Benson MD. Progression of transthyretin amyloid neuropathy after liver transplantation. Neurology. 2010;75(4):324-7.

30. Abramson N, Melton B. Leukocytosis: basics of clinical assessment. Am Fam Physician. 2000;62(9):2053-60.

31. Suhr O, Adams D, Coelho T, Conceicao I, Waddington CM, Schmidt H, et al. Further analysis of phase II trial of patisiran, a novel RNAi therapeutic for the treatment of familial amyloidotic polyneuropathy. XIVth International Symposium on Amyloidosis (ISA), Indianapolis, IN, USA; April 27-May 1, 2014: Abstract OP-72.

32. Adams D, Coelho T, Obici L, Merlini G, Mincheva Z, Suanprasert N, et al. Rapid progression of familial amyloid polyneuropathy: A multinational natural history study. Neurology 2015, Jul 24 [Epub ahead of print].

\section{Submit your next manuscript to BioMed Central and take full advantage of:}

- Convenient online submission

- Thorough peer review

- No space constraints or color figure charges

- Immediate publication on acceptance

- Inclusion in PubMed, CAS, Scopus and Google Scholar

- Research which is freely available for redistribution 